\title{
TENDENCIAS EMPRESARIALES MODERNAS Y SUS EFECTOS EN EL SISTEMA CONTRACTUAL
}

\section{TENDÊNCIASEMPRESARIAISMODERNASESEUSEFEITOSNOSISTEMACONTRATUAL}

\section{MODERN BUSINESS TRENDS AND THEIR EFFECTS ON THE CONTRACTUAL SYSTEM}

Licença CC BY:

Artigo distribuído sob os termos Creative Commons, permite uso e distribuição irrestrita em qualquer meio desde que o autor credite a fonte original.

\section{Juan Carlos Díaz Sánchez ${ }^{1}$}

\section{(c) (1)}

RESUMEN: En este artículo hablaremos de las tendencias empresariales modernas, cómo transforman el mercado y las relaciones empresariales entre proveedor y consumidor o usuario. Para luego analizar su impacto en el sistema contractual tradicional, evaluando la aparición del derecho al arrepentimiento, a la reflexión y la afectación a la libre voluntad contractual, así como pérdida del clásico consensualismo y la fuerza obligatoria del contrato.

PALABRAS CLAVE: Tendencias empresariales modernas, globalización, publicidad, ventas, tecnología, arrepentimiento, reflexión, libre arbitrio, buena fe.

RESUMO: Neste artigo falaremos sobre as tendências empresariais modernas e como elas transformam o mercado e as relações empresariais entre provedor e consumidor ou usuário. Vamos então analisar seus impactos no sistema contratual tradicional, analisando o surgimento do direito ao arrependimento, à reflexão e à manifestação da livre vontade contratual, assim como a perda do consensualismo clássico e da força de obrigação do contrato.

PALAVRAS-CHAVE: Tendências empresariais modernas, globalização, publicidade, vendas, tecnologia, arrependimento, reflexão, livre-arbítrio, boa-fé.

ABSTRACT: In this article, we talk about modern business trends, how the market is transformed, and the business relationships between supplier and consumer or user. We then analyze its impact on the traditional contractual system, evaluating the appearance of the right to repentance, reflection and affectation to the free contractual will, as well as the loss of classic consensualism and the mandatory force of the contract.

KEYWORDS: Modern business trends, globalization, advertising, sales, technology, regret, reflection, free will, good faith.

1 Abogado por la Universidad Nacional de Cajamarca. Doctorando en Derecho por la Pontificia Universidad Católica del Perú. Magíster en Derecho de la Empresa. Docente de la Escuela de Postgrado de la Universidad Nacional de Cajamarca y docente asociado en la Facultad de Derecho de la UPAGU. E-mail: jcdiazsanchez@yahoo.es 


\section{INTRODUCCIÓN}

Tradicionalmente se ha concebido al Contrato como "el acuerdo de dos o más partes para crear, regular, modificar o extinguir una relación jurídica patrimonial". Así lo establece el artículo $1351^{\circ}$ del Código Civil Peruano de 1984.

Sin embargo, los cambios culturales, económicos y tecnológicos que hoy vivimos son evidentes. Hemos sido abrazados y hasta absorbidos por fenómenos como la globalización, la tecnología de la información y hasta la dependencia de los grandes poderes económicos. Todo ello nos ha llevado a tener una sociedad basada en el consumismo (habiendo llegado al punto de creer que "felicitad" es sinónimo de "comprar") y el mercantilismo de las grandes empresas (quienes cada día quieren ganar más y más).

El mundo ha cambiado, y sigue cambiando. Las estructuras sociales y económicas se han transformado, generándose nuevas formas de hacer negocios y nuevos métodos para atraer y atrapar nuevos clientes. Recuerdo que cuando viajé por primera vez en avión, hace aproximadamente 20 años, para comprar el boleto tuve que tomar un taxi para buscar la oficina de la empresa aérea y esperar como treinta minutos para ser atendido, pues antes de mí había varias personas esperando. Pero ahora, sólo basta tener un celular a la mano y hacer la compra puede demorar tan solo tres minutos y sin salir de casa, o puedes hacerlo donde quiera que estés.

Lo antes mencionado nos lleva a preguntarnos: ¿Cómo han afectado al sistema contractual, las tendencias empresariales modernas?

\section{TENDENCIAS EMPRESARIALES MODERNAS}

Una de las áreas de la vida, que más cambios ha sufrido, es la actividad empresarial. Los empresarios están interesados en ampliar sus negocios y tener mayor participación en el mercado. Para ello, van creando nuevas formas de llegar a más lugares y nuevos métodos para captar más clientes, todo ello con el objetivo de obtener mejores utilidades y ser la empresa dominante del mercado.

Pero claro, estos objetivos no son sólo de algunos empresarios, sino parece ser un sentimiento dominante en el mundo empresarial y además respaldados por los gobiernos. De esta simbiosis de intenciones empresariales y gobiernos, van naciendo las tendencias empresariales modernas que van impactando en el sistema contractual.

A continuación, de manera breve mencionaremos aquellas tendencias empresariales que más impacto vienen causando en el sistema contractual. 


\subsection{LA GLOBALIZACIÓN}

Es producto del intenso interés de los empresarios y Estados, en romper las barreras fronterizas, logrando una mayor comunicación e interdependencia, teniendo como consecuencia la unificación de la sociedad y los mercados.

El resultado es la aparición de empresas transnacionales, que operan en diversos países y han logrado penetrar todas las esferas de la vida. Empresas que han logrado mayor poder económico que muchos Estados e incluso mayor influencia en la cultura y costumbres de las naciones, que sus propios gobiernos de turno (STAFFEN, 2015, p. 88). Y todo ello, como agentes externos, lo cual les da el privilegio de no asumir con todas las responsabilidades que generan sus actos y muchas veces saltarse las reglas jurídicas impuestas en los Estados en los que operan.

Incluso las empresas transnacionales han logrado la protección de sus inversiones a través de tratados internacionales, excluyéndose de la competencia jurisdiccional del país en donde operan, un ejemplo de ello es la creación del Centro Internacional de Arreglo de Diferencias Relativas a Inversiones (CIADI).

\subsection{MILLONARIA INVERSIÓN EN PUBLICIDAD}

Las empresas, con el pasar de los años, han aumentado su gasto en publicidad. No escatiman en contratar grandes empresas publicitarias, quienes incluso han comenzado a usar la psicología para lograr un solo objetivo: vender más. Y muchas veces no dudan incluso en manipular el inconsciente del ser humano, sólo con el objetivo de atraerlo y atraparlo en una supuesta oferta de algún producto.

A tal nivel va llegando la publicidad, que hoy se habla del "Neuromárketing", que estudia el comportamiento de los consumidores, creando técnicas que lleguen incluso a manipular los sentidos y emociones de los consumidores. Tal es su avance que países como España se han visto obligados a regular la llamada "publicidad subliminal".

Por ello, no es casualidad que Lionel Messi, uno de los mejores jugadores de la historia del fútbol, aparezca frecuentemente en anuncios publicitarios, al igual que Cristiano Ronaldo. Pues se trata de una estrategia más, el uso de los llamados "influencers"2. Y para ello, las empresas no escatiman en la inversión de miles de millones de dólares, al llegar al punto de que muchos deportistas ganan más por publicidad, que por el deporte mismo.

$2 \quad$ Un influencer es una persona que cuenta con cierta credibilidad sobre un tema concreto, y por su presencia e influencia en redes sociales puede llegar a convertirse en un prescriptor interesante para una marca. En: https://www.40defiebre.com/que-es/influencer (06.06.2019) 


\subsection{APARICIÓN DE NUEVAS MODALIDADES DE VENTA}

Como lo hemos manifestado, las empresas quieren vender más y más, pues quieren tener mayor participación en el mercado y con ello los socios logran mayores utilidades.

Para lograr tal objetivo: vender más. Las empresas han mejorado sus técnicas de venta, dando lugar a la aparición por ejemplo del "telemárketing" (venta por teléfono), "vending" (uso de máquinas expendedoras), "televenta" (venta por televisión"), "merchandising" (técnicas de presentación y gestión de productos).

El uso y combinación de todas las técnicas antes mencionadas, han dado como resultado lo que hoy conocemos como "ventas agresivas". Me da la impresión de que nos estamos convirtiendo en una jungla salvaje, en la cual nosotros los ciudadanos, estamos siendo cazados por los vendedores. Una muestra de ello es que recibamos llamadas por teléfono sin ningún tipo de restricciones, a cualquier hora y para recibir todo tipo de ofertas.

Y esto se agrava cuando las llamadas, trasgreden derechos fundamentales de las personas, como el derecho a la tranquilidad y a la verdad, pues muchos de los anuncios publicitarios que se reciben están llenas de medias verdades o información incompleta. Un ejemplo de esto se dio cuando recibí una llamada, en la cual me ofrecían un sofisticado programa de inglés, supuestamente garantizando convertirme en bilingüe en tan solo siete meses, sin asistir a clases y sin dar exámenes.

\subsection{USO DE LA TECNOLOGÍA}

La tecnología se ha convertido en un gran instrumento de desarrollo. Nos ha facilitado la vida y nos ahorra tiempo y espacio. Todos los días hacemos uso del internet, sea porque debemos enviar correos electrónicos o porque participamos activamente de las redes sociales como el Facebook.

Y claro, esto es aprovechado por las empresas, que han encontrado en la tecnología una gran oportunidad para facilitar y aumentar la celebración de contratos y con ello aumentar sus ventas. Es por ello que cada segundo somos bombardeados por publicidad o por ofertas. Gracias a la tecnología las empresas conocen nuestras preferencias en productos o servicios (conocen nuestro patrón de consumo), saben cuándo viajaremos y a dónde, saben si estamos buscando alguna oferta de servicio y saben las características de los productos que preferimos.

Y si a lo antes mencionado añadimos la casi nula protección de nuestros datos personales, entonces nos encontramos absorbidos por la publicidad que se orientan a lo que queremos contratar. 
Si un día hiciste una búsqueda de un vuelo en despegar.com, desde el siguiente segundo recibirás ofertas, las verás cuando abras tu correo electrónico, cuando navegues en redes sociales y hasta recibirás mensajes de texto en tu celular. Todo gracias a la maravilla de la tecnología.

Nos espera un futuro con profundas transformaciones en la economía, la sociedad, la cultura y las relaciones contractuales. Una muestra de ello es la aparición de la llamada "Tecnología 5G", que augura mayor velocidad y la conexión de todo con todo, a lo cual se viene llamando "el internet de las cosas".

\subsection{EL DINAMISMO Y CRECIMIENTO ECONÓMICO A REBALSADO EL CONTROL} ESTATAL

Los movimientos económicos se multiplican en segundos, las transacciones diarias son incontables y cada vez representan movimientos económicos de mayor envergadura. Esto ha puesto al descubierto Estados con instituciones débiles, sin capacidad para supervisar o controlar a las grandes empresas que operan desde el extranjero, y en consecuencia se vive la afectación a los derechos de los consumidores y/o usuarios.

Si a esto añadimos las grandes guerras comerciales, como la iniciada entre Estados Unidos y China. Cuya consecuencia es la desmedida imposición de aranceles e incluso la prohibición de venta de determinados productos (el caso Huawei es un ejemplo), irremediablemente afectará las relaciones contractuales de proveedores y sus clientes, generando inseguridad jurídica.

Pero además no olvidemos que vivimos en un mundo globalizado y en donde la tecnología es el soporte de nuestro trabajo y nuestras relaciones sociales y comerciales. Por lo tanto, una guerra comercial entre dos países con protagonismo en la economía mundial, en un ambiente de globalización y con predominio de la tecnología, indefectiblemente afectará las relaciones contractuales entre proveedores y clientes.

\section{EFECTOS EN EL SISTEMA CONTRACTUAL}

Ahora corresponde identificar y analizar los efectos que las tendencias empresariales modernas causan en el sistema contractual tradicional. 


\subsection{EL DERECHO DE ARREPENTIMIENTO FRENTE AL PRINCIPIO DEL CONSENSUALISMO Y FUERZA OBLIGATORIA DEL CONTRATO}

El profesor francés Malinvaud P. (1997), habla sobre el Derecho de Arrepentimiento, manifestando que: "La gran ola de derechos de retractación no apareció sino en los años 1970 y se sitúa en el gran movimiento de protección de los consumidores" (p. 64). El autor hace referencia al derecho que todo consumidor tiene de retirarse de un contrato o resolverlo, dentro de un cierto plazo.

No cabe duda que este derecho de arrepentimiento, entra en conflicto con principios tradicionales del sistema contractual, como el consensualismo y la fuerza obligatoria del contrato. El primer principio hace referencia al consenso o acuerdo entre las partes, como elemento consumador de un contrato, sin que se exija estrictas formalidades. Por su parte, con la fuerza obligatoria del contrato, se consideraba al contrato como un ente inmutable, incluso con la fuerza necesaria para afrontar al Estado mismo.

Sin embargo, la realidad ha cambiado, pues hoy el derecho de arrepentimiento encuentra plena justificación. En una sociedad dominada por el consumismo, la avasalladora publicidad que nos imponen los proveedores y la demoledora estrategia para "enganchar clientes", nos hace pensar en incorporar a las legislaciones el derecho de todo consumidor o usuario a decidir, dentro de un plazo prudencial, dejar sin efecto un contrato.

Es frecuente, en el Perú se puede recibir hasta cinco llamadas telefónicas diarias, de instituciones financieras o empresas aseguradoras, ofreciendo "grandes beneficios" si se acepta una tarjeta de crédito o un seguro. Obviamente cada llamada está preparada para lograr un contrato, desde la persona que habla al teléfono, la hora en que llaman (la más inoportuna posible), la forma como te explican los supuestos beneficios e incluso la insistencia ante la negativa.

Estas nuevas circunstancias precontractuales, que han aparecido gracias al uso de la tecnología y los nuevos métodos de venta, nos obligan a pensar en la necesidad de incorporación en el sistema contractual moderno, el derecho de arrepentimiento. Ello nos lleva a concluir, que un contrato debe dejarse sin efecto, no sólo por la existencia de una cláusula abusiva, sino también porque en el origen del mismo (etapa de negociación), se afectó derechos del consumidor o usuario, porque el abuso estuvo en la forma como se atrajo al cliente, sea por el uso de métodos poco transparentes o por el uso de información incompleta y hasta falsa información. 


\subsection{EL DERECHO A LA REFLEXIÓN}

El derecho al arrepentimiento, nos lleva a pensar en el "Derecho a la Reflexión". Entendido éste como el derecho que todos debemos tener, de analizar, evaluar, pensar y repensar, si fuera necesario, antes de decidir asumir una obligación contractual, y todo ello en un ambiente adecuado.

Este derecho a la reflexión, tendría su justificación en la abrumadora publicidad y ofertas que recibimos hoy, por todos los medios que tenemos a nuestra disposición, sea teléfono, redes sociales, WhatsApp, correos electrónicos, etc. Sin dejar de considerar la agresividad en las ventas, al punto que ahora se escribe mucho sobre "las ventas agresivas". Sumamos a ello, el uso desmedido y sin limitaciones, de nuestros datos personales por parte de los proveedores, quienes se transfieren entre sí nuestros números de celular privados, cuentas de redes sociales y correos electrónicos. Y no podemos dejar de mencionar la facilidad que se tiene hoy para perfeccionar un contrato, pues basta un "clic" para asumir obligaciones contractuales que inmediatamente se van cargando a nuestras cuentas bancarias.

Al considerar esta nueva realidad, el respeto al derecho a la reflexión, vendría a representar un límite a la actuación de los proveedores de productos y servicios. Esto significa que, una de las grandes tareas del derecho contractual hoy, es dar contenido al derecho a la reflexión, contenido que servirá para establecer los límites a la publicidad y ofertas de los vendedores, y que además constituya el fundamento para establecer el derecho al arrepentimiento, esto es, el derecho a resolver unilateralmente un contrato.

La justificación práctica para lo antes mencionado, la podemos encontrar en la forma como los bancos han comenzado a ofertar los seguros de las tarjetas de crédito que ellos mismos emiten. En el Perú es común que, cuando vas a realizar alguna operación en alguna ventanilla de un banco, quien te atiende, te ofrezca un seguro para tu tarjeta de crédito e incluso un seguro de vida (pues ahora las empresas bancarias también son dueñas de empresas de seguros). Esto evidencia cómo los bancos están usando todas las herramientas que tienen en sus manos para lograr tal venda: el lugar más inoportuno e inadecuado para reflexionar sobre los alcances de un contrato de seguros (como la ventanilla de un banco, cuando estás apurado y ves a la gente esperando su turno), las personas idóneas (quien te está dando un servicio y con ello parece hacerte un favor, al que debes retribuir).

A mi consideración, hemos pasado de la negociación de un contrato, a "la venta de un contrato". Hoy ya no se negocian los contratos, pues por la premura del tiempo, ni siquiera podemos conocer todas las condiciones a las que nos sometemos y menos aún tenemos la posibilidad de sugerir un cambio. Hoy, el contrato es un producto más que es objeto de múltiples ofertas. 


\subsection{LA LIBRE VOLUNTAD FRENTE A LA IMPOSICIÓN CONTRACTUAL}

En una economía globalizada, con una abrumadora publicidad y el uso de ventas cada vez más agresivas, nos preguntamos: ¿Aún existe la libre voluntad en la formación de un contrato y para decidir con quién contratar? ¿O es que el sistema económico moderno nos está imponiendo los bienes y servicios, y en consecuencia nuestra libre manifestación de la voluntad ha sido anulada?.

Tales preguntas me surgieron producto de una experiencia laboral. En el 2019 enseñé en la Facultad de Derecho de una universidad en la hermosa ciudad de Cajamarca, Perú. Como no informé oportunamente el banco en donde tenía mi "cuenta sueldo" (cuenta de ahorros destinada a recibir el pago de remuneraciones), la universidad decidió depositar mi sueldo en el Banco Interbank. Sin embargo, como yo no era cliente de este banco, para poder retirar el dinero me dijeron que debía firmar un contrato de apertura de cuenta sueldo, que incluía la entrega de una tarjeta de débito y pretendieron hacerme firmar un contrato de adelanto sueldo. Pero yo tenía una cuenta sueldo en otro banco y también una tarjeta de débito, por lo que, no necesitaba tales servicios. Y fue esto lo que les hice saber a los empleados del banco, pero condicionaron el retiro de mi dinero a la firma y recepción de los contratos antes mencionados. Sentí que me imponían la firma de contratos y que no les importaban mi libre voluntad. Situaciones como estas son cada vez más frecuentes, pues las empresas aprovechan la mínima oportunidad para atrapar nuevos clientes, pues en el mercado de hoy, se libra diariamente una batalla por atrapar nuevos clientes. Y claro que podía demandar judicialmente al banco, pero esto demandaría gasto de recursos económicos y tiempo.

Otro ejemplo en el Perú es la facultad que tiene la empresa Telefónica del Perú, para modificar unilateralmente las condiciones contractuales firmadas con sus usuarios. Y de manera muy particular, incluso puede subir unilateralmente el costo de las tarifas, previa autorización del órgano estatal competente (en el caso peruano es Osiptel) (SUMARRIVA, GRADOS, ARREDONDO, 2013, p. 44). Es decir, en el Perú es posible que un ciudadano haya firmado un contrato, obligándose a pagar un precio determinado, pero no será necesario que exprese su voluntad para que el precio sea aumentado, pues la empresa puede aumentarlo unilateralmente, sólo requiriendo la autorización de la entidad reguladora (Osiptel). Es por eso que el 14 de febrero del 2020, el Organismos Supervisor de Inversión Privada en Telecomunicaciones (Osiptel), ordenó a Telefónica del Perú S.A.A cancelar el aumento unilateral de las tarifas de internet fijo, pues tales modificaciones contractuales requerían, no la aprobación del usuario, sino del Osipte. Situaciones como las detalladas, nos llevan a la conclusión de que, en la moderna economía de mercado, son más frecuentes las situaciones en las cuales la libre voluntad está prácticamente anulada, y los contratos o las modificaciones a los mismos, nos son impuestos. 


\subsection{DE LOS CONTRATOS POR ADHESIÓN A LA MANIPULACIÓN DEL COMPORTAMIENTO}

El sistema contractual nació vinculado a dos pilares fundamentales, la libre voluntad y la fuerza obligatoria de los contratos. El primer pilar significaba que las personas tenían el derecho de decidir con quién contratar y bajo qué condiciones hacerlo. Pero luego, el crecimiento económico, producto de la revolución industrial y la movilización de grandes capitales, vio la necesidad de facilitar la contratación, ahorrando costos de transacción y haciendo partícipes a grandes masas de personas. Para ello surgió los contratos por adhesión, que estaban diseñados por una de las partes y puestas a consideración de la otra para que acepte o no celebrarlo.

No cabe duda que la aparición de los contratos por adhesión, representó una seria afectación a uno de los pilares fundamentales del sistema contractual, esto es, la libre voluntad. Pues ésta se redujo a aceptar o no un contrato.

Pero una vez más, la globalización, el uso de la tecnología, las nuevas técnicas de ventas y las millonarias inversiones en publicidad, nos muestran que las empresas están decididas a dar un paso más allá (PAFFARINI, 2015). En mi opinión las empresas pretenden llegar a la manipulación del comportamiento, pues ellas quieren decidir cuándo, qué y en dónde compramos, incluso pretenden decidir cuánto pagamos.

Una muestra de lo manifestado, es el uso indiscriminado de nuestros datos personales, y por supuesto, la poca o nula protección de los mismos por parte del Estado. Si un día usaste un buscador en internet para ver el precio de un hotel para unas vacaciones, te aseguro que al segundo te llegarán múltiples y variadas ofertas de hoteles, para el lugar que estás buscando y en las fechas que deseas.

Pero ello no es todo, hay empresas que, con el atractivo de ofertas, para lograr el beneficio de las mismas, te piden el número de tu documento de identidad en cada compra, supuestamente para que vayas acumulando puntos para futuras ofertas. Pero en realidad, gracias al uso de la tecnología, la empresa está conociendo el patrón de consumo de sus clientes, lo cual les permitirá orientar sus ofertas a las necesidades más frecuentes.

Otro ejemplo de lo manifestado es lo que me tocó vivir en una compra en una cadena farmacéutica (Inkafarma). Hice una compra y luego de pagar, quien me atendió me manifestó que había sido elegido para participar de un sorteo de S/. 5,000 soles (aproximadamente US\$ 1,500 dólares americanos). Para ello debía llenar un cupón y luego de firmarlo debía depositarlo en la misma empresa para el sorteo. Sin embargo, cuando leí el cupón quedé asombrado, pues en realidad estaba firmando una autorización para el uso de mis datos personales, incluso autorizando que tales datos sean vendidos al extranjero. 
¿Por qué las empresas están tan interesadas en tener tus datos personales como número de celular y correo electrónico? ¿por qué están tan interesadas en conocer tus gustos y preferencias a través del patrón de consumo? Una sola respuesta me viene a la mente, pretenden manipular nuestras preferencias (y con ello estarían manipulando nuestro comportamiento). Para ello, buscan que sus ofertas sean más eficaces, orientadas a nuestras necesidades. Las empresas van acumulando y organizando toda la información que puedan obtener de sus clientes, pues esta información les servirá no sólo para diseñar sus productos sino también para saber cuánto se está dispuesto a pagar por un producto, con ello, tienen todas las herramientas para lograr el máximo beneficio económico posible (PAFFARINI, 2015, p. 45).

Ahora me pregunto con más frecuencia si lo que compro, es porque yo lo decidí o porque los vendedores lo decidieron por mí. Es decir, realmente ejercí mi libre voluntad o se trató simplemente de un comportamiento inducido, producto de la publicidad, del neuromárketing o de la publicidad subliminal. No olvidemos que una de las tendencias empresariales modernas, es el uso cada vez más intenso de la psicología del consumidor y de los patrones de consumo.

\section{5. ¿AÚN EXISTE LA BUENA FE CONTRACTUAL?}

La buena fe contractual es otro de los pilares fundamentales del sistema contractual. Es fundamental para generar confianza en los negocios y construir economías sólidas y saludables. Pero este elemento tradicionalmente fundamental, también se ha visto afectado por las tendencias empresariales modernas.

El objetivo de atrapar nuevos clientes y ampliar la cartera de servicios, ha llevado a las empresas a contratar personal que sólo se dediquen a ofertar sus servicios, principalmente por llamadas telefónicas. Pero el pago por tales servicios se hace en base a comisiones, es decir, sus ganancias dependerán de la cantidad de clientes que capten, a mayor número de clientes, mayor remuneración. Se genera así una motivación para "hacer todo lo que sea necesario" para captar clientes.

Entonces la buena fe de una de las partes (el proveedor), se convierte en mala fe, pues como parte de ese "hacer todo lo que sea necesario" para captar clientes, se traduce en información incompleta dada al cliente o incluso falsa información. Todo con el único objetivo de ganar un cliente más. En esta jungla salvaje, de competencia entre empresarios, en donde parece que "todo vale", es cada vez más frecuente encontrar a empresas denunciadas por sus clientes porque no les dieron la información completa o porque recibieron información que nos e ajusta a la verdad. 
No digo que en el mercado moderno se actúe con mala fe, digo que es cada vez más frecuente que los empresarios hagan uso de la mala fe (traducido en falta de transparencia en los negocios y falta de veracidad en sus ofertas), para tener mayor participación en el mercado. Esto nos debe llevar a idear nuevas fórmulas de protección a los consumidores o usuarios, que sean efectivos y eficientes. Pero además, en determinados casos y bajo determinadas circunstancias, incluso quizá debamos llegar a presumir la mala fe de los proveedores, y con ello crear sanciones económicas que puedan corregir inmediatamente la mala actuación en el mercado.

Parece que estamos en una guerra sin fin, en donde el vendedor quiere extraer el máximo recurso posible de sus clientes, sin importar las formas o métodos. Todo vale con tal de lograr la máxima rentabilidad posible, parece que este es el pensamiento del empresario hoy. Más de una vez he pensado que la ley de la oferta y la demanda (principio rector del libre mercado), ha sido diseñada sólo para lograr extraer el máximo recurso posible de los consumidores o usuarios. Pero esta ley no tiene un factor o elemento que ponga límite a la avaricia del empresario y logre un real y verdadero equilibrio en la confluencia de la oferta y la demanda.

Si bien las modernas tendencias empresariales han traído grandes beneficios y oportunidades, también trajeron grandes riesgos y desigualdades. Por ello coincidimos con lo manifestado por Tapscott y Caston, quienes al hablar de los cambios empresariales, dijeron que: "Hay nuevas oportunidades, pero también existen peligros potenciales para las naciones, las culturas, las economías y las personas" (Tapscott \& Caston, 1995)

\section{CONCLUSIONES}

3.1.- En la actividad empresarial moderna, un contrato debe dejarse sin efecto no sólo por las condiciones abusivas expresadas en el contrato (llamadas cláusulas abusivas), sino también cuando el abuso es identificado en la etapa pre contractual (la negociación). Estas condiciones abusivas pueden reflejarse en la forma como se atrajo al cliente, quizá por el uso de métodos poco transparentes, uso de información incompleta y hasta falsa información.

3.2.- El derecho al arrepentimiento y el derecho a la reflexión, surgen como instrumentos a favor del consumidor o usuario, para contrarrestar la abrumadora ola de publicidad y ofertas, así como los métodos de venta modernos, cuya agresividad exigen una eficiente protección de la parte débil en una relación contractual.

3.4.- Las tendencias empresariales modernas, nos han llevado de la negociación de un contrato, a "la venta de un contrato". Ya no se negocian los contratos, sino se venden, como si fuera cualquier producto del mercado. 
3.5.- Quizá, en el sistema contractual actual, en vez de tener a la buena fe como regla, habría que pensar en la actuación de mala fe de quienes tienen un status de poder y que bajo determinadas circunstancias, actúan en el mercado con el único objetivo de lograr las mayores ganancias posibles, sin importar el derecho de los usuarios o consumidores.

3.4.- Dadas las tendencias empresariales modernas, ¿será que la ley de la oferta y la demanda (principio rector del libre mercado), hoy es usado por los grandes empresarios para lograr extraer el máximo recurso posible de los consumidores o usuarios? Si así fuera, tenemos la tarea de diseñar una nueva ley o principio económico o contractuales, que ponga límite a la avaricia del empresario y logre un real y verdadero equilibrio en las relaciones contractuales.

\section{BIBLIOGRAFÍA}

MALINVAUD, P. (2007). Droit des obligations, 10th ed., LexisNexis, Paris.

PAFFARINI, J. (2015). Modelli costituzionali e società di mercato. Lima, Perú. EGACAL.

STAFFEN, M. R. (2015). Globalismo jurídico. Traducción de Guido César Grados. Lima, Perú. San Marcos.

SUMARRIVA, A. C.; GRADOS, G. A; ARREDONDO, V. (2013). La constitucionalización del derecho en el Perú. Lima, Perú. EGACAL.

TAPSCOTT, D. y CASTON, A. (1995) Cambio de Paradigmas Empresariales. Bogotá, Colombia. McGraw-Hill Interamericana. TORRES, M. (1997). Nuevas Tendencias del Derecho Contractual: Libro Homenaje a Manuel De la Puente y Lavalle. Lima, Perú. Grijley.

RECEBIDO EM: 12/08/2019

APROVADO EM: 21/11/2019 